\title{
Curriculum CycleGAN for Textual Sentiment Domain Adaptation with Multiple Sources
}

\author{
Sicheng Zhao* ${ }^{*}$ \\ schzhao@gmail.com \\ University of California, Berkeley \\ USA \\ Xiangyu Yue ${ }^{\dagger}$ \\ xyyue@berkeley.edu \\ University of California, Berkeley \\ USA
}

\author{
Yang Xiao ${ }^{\dagger}$ \\ 17822018007@163.com \\ Nankai University \\ China
}

\author{
Jufeng Yang \\ yangjufeng@nankai.edu.cn \\ Nankai University \\ China
}

\author{
Jiang $\mathrm{Guo}^{\dagger}$ \\ jiang_guo@csail.mit.edu \\ Massachusetts Institute of Technology \\ USA \\ Ravi Krishna \\ ravi.krishna@berkeley.edu \\ University of California, Berkeley \\ USA
}

\author{
Pengfei Xu \\ xupengfeipf@didiglobal.com \\ Didi Chuxing \\ China
}

\author{
Kurt Keutzer \\ keutzer@berkeley.edu \\ University of California, Berkeley \\ USA
}

\begin{abstract}
Sentiment analysis of user-generated reviews or comments on products and services in social networks can help enterprises to analyze the feedback from customers and take corresponding actions for improvement. To mitigate large-scale annotations on the target domain, domain adaptation (DA) provides an alternate solution by learning a transferable model from other labeled source domains. Existing multi-source domain adaptation (MDA) methods either fail to extract some discriminative features in the target domain that are related to sentiment, neglect the correlations of different sources and the distribution difference among different sub-domains even in the same source, or cannot reflect the varying optimal weighting during different training stages. In this paper, we propose a novel instance-level MDA framework, named curriculum cycle-consistent generative adversarial network (C-CycleGAN), to address the above issues. Specifically, C-CycleGAN consists of three components: (1) pre-trained text encoder which encodes textual input from different domains into a continuous representation space, (2) intermediate domain generator with curriculum instance-level adaptation which bridges the gap across source and target domains, and (3) task classifier trained on the intermediate domain for final sentiment classification. C-CycleGAN transfers source samples at instance-level to an intermediate domain that is closer to the target domain with sentiment semantics preserved and without losing discriminative features. Further, our dynamic instance-level weighting mechanisms can assign the optimal weights to different
\end{abstract}

\footnotetext{
${ }^{*}$ Corresponding author.

$\dagger$ Equal contribution
}

This paper is published under the Creative Commons Attribution 4.0 International (CC-BY 4.0) license. Authors reserve their rights to disseminate the work on their personal and corporate Web sites with the appropriate attribution.

WWW'21, April 19-23, 2021, Ljubljana, Slovenia

(c) 2021 IW3C2 (International World Wide Web Conference Committee), published under Creative Commons CC-BY 4.0 License.

ACM ISBN 978-1-4503-8312-7/21/04.

https://doi.org/10.1145/3442381.3449981 source samples in each training stage. We conduct extensive experiments on three benchmark datasets and achieve substantial gains over state-of-the-art DA approaches. Our source code is released at: https://github.com/WArushrush/Curriculum-CycleGAN.

\section{CCS CONCEPTS}

- Information systems $\rightarrow$ Sentiment analysis; • Computing methodologies $\rightarrow$ Natural language processing; Transfer learning.

\section{KEYWORDS}

Domain adaptation, multiple sources, sentiment analysis, cycleconsistent generative adversarial network, curriculum learning

\section{ACM Reference Format:}

Sicheng Zhao, Yang Xiao, Jiang Guo, Xiangyu Yue, Jufeng Yang, Ravi Krishna, Pengfei Xu, and Kurt Keutzer. 2021. Curriculum CycleGAN for Textual Sentiment Domain Adaptation with Multiple Sources. In Proceedings of the Web Conference 2021 (WWW'21), April 19-23, 2021, Ljubljana, Slovenia. ACM, New York, NY, USA, 12 pages. https://doi.org/10.1145/3442381.3449981

\section{INTRODUCTION}

The wide popularity of social networks and mobile devices enables human beings to reflect and share their opinions of the products and services they purchase online using text, images, and videos [13, 23, $24,42,84,88,90,91]$. For example, when we plan to buy something, it is of high probability that we take a look at the comments on what others feel about this product. If the negative comments dominate the feedback, we might change our minds to a different brand. Sentiment analysis of user-generated large-scale multimedia data can not only help the customers to select what they want, but also prompt enterprises to improve the quality of their products and services $[11,90]$. Among different multimedia modalities, text, the one focused on in this paper, is the most direct and popular one [13]. 


\begin{tabular}{cc}
\hline & Accuracy on target \\
\hline Single-best DANN & 69.2 \\
\hline Source-combined DANN & 67.0 \\
\hline C-CycleGAN & 73.8 \\
\hline
\end{tabular}

(a) Quantitative comparison

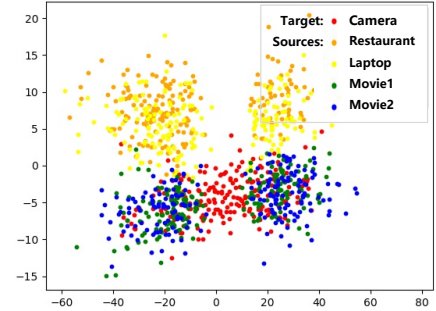

(b) Before adaptation

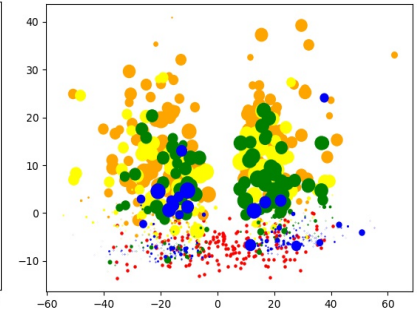

(c) After adaptation
Figure 1: An example of domain shift in the multi-source scenario on the Reviews-5 dataset [79], where Camera (red points) is set as the target domain and the rest as source domains. (a) Naively combining multiple sources into one source and directly performing single-source domain adaptation (DANN [19]) does not guarantee better performance compared to just using the best individual source domain (69.2 vs. 67.0). The proposed C-CycleGAN framework achieves significant performance improvements over the source-trained model baselines (73.8 vs. 69.2). (b) and (c) visualize the representation space before and after adaptation. We can see clear domain shift across the sources and the target. After our domain adaptation, the source samples that are closer to the target domain (smaller points) are better aligned to the target domain (larger points indicate smaller sample weights).

Recent studies [3, 11, 20, 35, 39, 69, 70, 76, 82] have shown that deep neural networks (DNNs) achieve the state-of-the-art performance on textual sentiment analysis. However, training a DNN to maximize its capacity usually requires large-scale labeled data, which is expensive and time-consuming to obtain. One alternate solution is to train a DNN on a labeled source domain and transfer the DNN to the target domain. However, due to the presence of "domain shift" [63], i.e. the distribution differences between the source and target domains, direct transfer may result in significant performance degredation [29, 65, 77, 80]. Domain adaptation (DA) $[36,47,58,85,92]$ that aims to minimize the impact of domain shift provides an alternate solution by learning a model on the source domain with high transferability to the target domain.

Current DA methods for textual sentiment analysis mainly focus on the single-source unsupervised setting $[40,72]$, i.e. in which there is only one labeled source domain and one unlabeled target domain. While these unsupervised domain adaptation (UDA) methods perform well when the domain gap between the source and target domains is relatively small, they may fail when the domain gap is large or when there are multiple labeled source domains [26, 85], which is a more practical scenario. For example, if we have a target Kitchen domain, which may include reviews on cookbooks, bowls, and electric kettles, and three source domains, books, cookware, and electronics, it is difficult to perfectly align each source and the target. Naive combination of different sources into one source and direct application of single-source UDA algorithms may lead to suboptimal results, because domain shift also exists across different sources, as shown in Figure 1. Sufficiently exploiting complementary information from different sources can allow for learning a better representation for the target domain, which calls for effective multi-source domain adaptation (MDA) techniques [58, 85].

Recently, some deep MDA approaches have been proposed for textual sentiment classification, most of which are based on adversarial learning, containing a pair of feature extractors and domain classifier (e.g. MDAN [83], MoE [26]). These methods mainly focus on extracting domain-invariant features of different domains, aligning each source and the target separately, or assigning weights to the source samples statically. Although they can obtain domaininvariant features among different domains, there are still some limitations. First, some discriminative features in the target domain that are related to sentiment might be missing. Since the shared feature extractor mainly aims to extract domain-invariant features by projecting both source samples and target samples to a lower-dimensional space, it may not include all sentiment-related features in the target domain. Second, some existing MDA methods separately align each source and the target and then combine the prediction results with known domain labels, which neglects the correlations of different source domains and different sub-domains even in each source. These methods would naturally fail when the domain labels of labeled source samples are not available. Finally, existing sampling-based methods mainly focus on selecting source samples that are closer to the target by training source selection models to calculate the weight of each sample (e.g. MDDA [89], CMSS [77]), which cannot reflect the varying optimal weighting during different training stages.

In this paper, we propose a novel instance-level multi-source domain adaptation framework, named curriculum cycle-consistent generative adversarial network (C-CycleGAN), to address the above issues for textual sentiment classification. First, in order to encode all text instances in both source and target domains into a latent continuous representation space with minimal information loss, we introduce text reconstruction to better preserve information. Second, for the encoded source representations, we generate an intermediate domain to align the mixed source and target domains using a generative adversarial network (GAN) with cycle-consistency. To explore the importance of different source samples in a batch, we assign weights to them at instance-level with novel dynamic model-based and model-free weighting mechanisms. Finally, based on the adapted representations and corresponding source sentiment labels, we train a transferable task classifier. The sentiment loss of the classifier is also backpropagated to the source-to-target generator to preserve the sentiment information before and after generation. Extensive experiments are conducted on three benchmark datasets: Reviews-5 [79], Amazon benchmark [7], and Multilingual Amazon Reviews Corpus [8]. The results show that the proposed C-CycleGAN significantly outperforms the state-of-theart DA methods for textual sentiment classification.

In summary, the contributions of this paper are threefold: 
(1) We propose a novel MDA method, named curriculum cycleconsistent generative adversarial network (C-CycleGAN), to minimize the domain shift between multiple source domains and the target domain. To the best of knowledge, we are the first to generate an intermediate representation domain with cycle-consistency and sentiment consistency for textual sentiment adaptation.

(2) We design novel instance-level model-based and model-free weighting mechanisms, which can update the sample weights dynamically. In this way, our framework does not require domain labels of samples, which allows it to exploit complementary information of all labeled source samples from different domains.

(3) We conduct extensive experiments on three benchmark datasets As compared to the best baseline, the propsoed C-CycleGAN achieves $1.6 \%, 1.2 \%$, and $13.4 \%$ improvements in average classification accuracy on Reviews-5, Amazon benchmark, and Multilingual Amazon Reviews Corpus, respectively.

\section{RELATED WORK}

Textual Sentiment Analysis. Textual sentiment analysis, or opinion mining, aims to assess people's opinions, emotions, and attitudes from text towards entities such as products, services, or organizations [81]. The wide popularity of social networks such as product reviews, forum discussions, and WeChat, contributes to the rapid development of this task $[11,81]$. Traditional sentiment analysis methods mainly focused on designing hand-crafted features [44, 46], which are fed into standard classifiers, such as SVM. Recent efforts on sentiment analysis are mainly based on DNNs [69, 81], which have shown great success in many natural language processing tasks. Some typical deep models that have been applied to sentiment analysis include Recursive Auto Encoder [16, 49, 53], Recursive Neural Tensor Network [54], Recurrent Neural Network (RNN) [62], Long short-term memory (LSTM) [27], Tree-LSTMs [61], RNN Encoder-Decoder [12], and BERT [14]. The above supervised learning methods usually require a large volume of labeled data for training $[11,40]$. However, high-quality sentiment labels are often labor- and time-consuming to obtain. In this paper, we employ a Bi-LSTM [27] as encoder and a multi-layer perceptron as classifier for the sentiment classification adaptation task.

Single-source UDA. Recent single-source UDA (SUDA) methods mainly employ deep learning architectures with two conjoined streams [93, 95]. One is trained on the labeled source data with a traditional task loss, such as cross-entropy loss for classification. The other aims to align the source and target domains to deal with the domain shift problem with different alignment losses, such as discrepancy loss, adversarial loss, self-supervision loss, etc. Discrepancy-based methods employ some distance measurements to explicitly minimize the discrepancy between the source and target domains on specific activation layers, such as maximum mean discrepancies $[41,68,72]$, correlation alignment $[55,56,95]$, and contrastive domain discrepancy [33]. Adversarial discriminative models usually employ a domain discriminator to adversarially align the extracted features between the source and target domains by making them indistinguishable $[10,19,32,37,51,64,66,71]$. Besides the domain discriminator, adversarial generative models also include a generative component to generate fake source or target data typically based on GAN [25] and its variants, such as CoGAN [38], SimGAN [52], and CycleGAN [29, 87, 94]. Selfsupervision based methods incorporate auxiliary self-supervised learning tasks into the original task network to bring the source and target domains closer. The commonly used self-supervision tasks include reconstruction $[9,21,22]$, image rotation prediction [60, 73], jigsaw prediction [5], and masking [67]. Although these methods achieve promising results for SUDA tasks, they suffer from significant performance decay when directly applied to MDA task.

Multi-source Domain Adaptation. Based on some theoretical analysis [2, 28], multi-source domain adaptation (MDA) aims to better deal with the scenario where training data are collected from multiple sources $[58,86]$. The early shallow MDA methods mainly include two categories [58]: feature representation approaches [6, $17,18,57]$ and combination of pre-learned classifiers [59, 75]. Some special MDA cases are considered in recent shallow methods, such as incomplete MDA [15] and target shift [50].

Recently, some representative deep learning based MDA methods are proposed, such as multisource domain adversarial network (MDAN) [83], deep cocktail network (DCTN) [74], Mixture of Experts (MoE) [26], moment matching network (MMN) [48], multisource adversarial domain aggregation network (MADAN) [86], multi-source distilling domain adaptation (MDDA) [89], and curriculum manager for source selection (CMSS) [77]. MDAN, DCTN, MoE, MMN, MADAN, and MDDA all require domain labels of source samples. MDDA and CMSS select source samples that are closer to the target domain with a static weighting mechanism, while the others do not consider the importance of different source samples. The MDA methods for textual sentiment classification, e.g. MDAN and MoE, only focus on extracting domain-invariant features, which may lose discriminative features of the target domain that are related to sentiment. Different from these methods, for the source samples, we generate an intermediate domain that is closer to the target domain with cycle-consistency and sentiment consistency. Further, we propose novel dynamic instance-level weighting mechanisms to assign weights to the source samples without the requirement of domain labels.

\section{PROPOSED APPROACH}

In this section, we formally define the MDA problem, give an overview of the proposed Curriculum CycleGAN (C-CycleGAN) framework, present each component of C-CycleGAN in detail, and finally introduce the joint learning process.

\subsection{Problem Definition}

We consider the multi-source unsupervised domain adaptation setup for textual sentiment classification, under the covariate shift assumption [47]. Assuming access to $k$ source domains with labeled training data, denoted by $\left\{\mathcal{S}_{i}\right\}_{i=1}^{k}$, where each domain $\mathcal{S}_{i}$ contains a set of examples drawn from a joint distribution $p^{\left(S_{i}\right)}(\mathrm{x}, \mathrm{y})$ on the input space $\mathcal{X}$ and the output space $\mathcal{Y}$, we seek to learn a sentiment classifier $f: \mathcal{X} \rightarrow \mathcal{Y}$ that is transferable to a target domain $\mathcal{T}$, where only unlabeled data is available.

\subsection{Overview}

Our model bridges the domain gap by generating an intermediate domain using CycleGAN [94] trained with a learned curriculum 


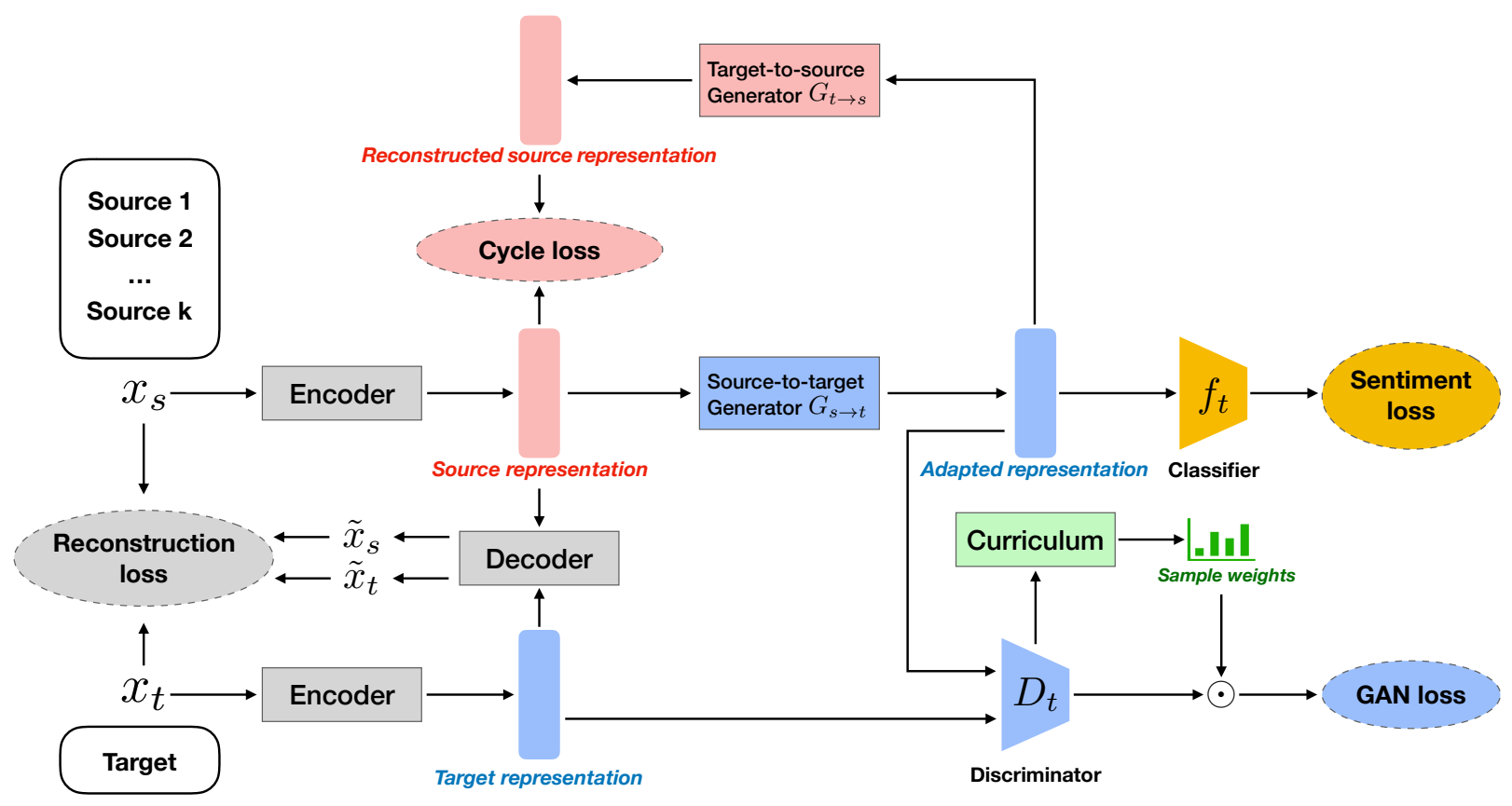

Figure 2: Illustration of the proposed C-CycleGAN framework. A text encoder is first pre-trained with a reconstruction loss to encode all text instances from the source and target domains into a latent continuous representation space (gray). Then the model is jointly trained using the cycle-consistency loss (pink), the curriculum GAN loss (blue), and the sentiment classification loss (yellow). We depict here the model-free curriculum (green) for sample weighting.

(C-CycleGAN). As shown in Figure 2, the proposed framework has three primary components:

Pre-trained Text Encoder: Encode texts from source and target domains into a semantic-preserving latent continuous representation space $\mathcal{Z}$. This module is pre-trained using a seq2seq-based text autoencoder in an unsupervised fashion.

Intermediate Domain Generator: Generate an intermediate domain to align the multiple sources and the target. At the core of this component is a curriculum cycle-consistent generative adversarial network, which employs a domain adversarial loss for distributional alignment, and use cycle-consistency to prevent mode collapse. To deal with the varied relevance of the mixed-source instances to the target domain at a specific training stage, we learn a curriculum to dynamically assign weights to source samples based on their proximity to the target domain distribution.

Task Classifier: Train the sentiment classifier based on the adapted representations in the intermediate domain and corresponding sentiment labels in the source domains.

\subsection{Pre-trained Text Encoder}

We use seq2seq-based text reconstruction to pre-train our text encoder, in order to obtain a semantic-preserving latent representation space. Let $x$ denote a sequence of tokens $w_{1}, \ldots, w_{L}$, where $L$ is the sequence length. The reconstruction process can be summarized as the following encoding-decoding paradigm:

$$
\mathbf{z}=\operatorname{Enc}(x ; \boldsymbol{\theta}) ; \tilde{x}=\operatorname{Dec}(\mathbf{z}, x ; \boldsymbol{\psi})
$$

where $\mathbf{z}$ is the text representation. We use a bidirectional LSTM (Bi-LSTM) [27] as the encoder, and obtain the representation $\mathbf{z}$ of an input sequence by concatenating the last states of forward LSTM and backward LSTM. A unidirectional LSTM then reconstructs $x$ autoregressively conditioned on $\mathbf{z}$. At each time step of generation, we randomly sample from the ground-truth token and the generated token as input for the next token prediction. The overall reconstruction loss over both source and target domain data can thus be written as:

$$
\mathcal{L}_{\text {rec }}=\mathbb{E}_{x \sim X_{S} \cup \mathcal{X}_{T}}\left[-\frac{1}{L} \sum_{t=1}^{L} \log P\left(\tilde{x}_{t} \mid x_{<t}, \tilde{x}_{<t}, \mathbf{z}\right)\right]
$$

After pre-training, the encoder will be fixed and the encoded representations will be directly used for the generation of the latent intermediate domain (Section 3.4).

Alternatively, we can directly use publicly available text encoders like BERT [14], which are designed to be general-purpose and pretrained in a self-supervised fashion on a mixture of data sources. In this study, we experiment with BERT, and take the hidden state of the "[CLS]" token as the text representation. ${ }^{1}$

\subsection{Intermediate Domain Generator}

GAN with Cycle-consistency. This module generates an intermediate representation domain from the pre-trained representation space $\mathcal{Z}$ to bridge the gap across source and target, as shown in

\footnotetext{
${ }^{1}$ Note that the "[CLS]" representation is typically used as a text representation at the fine-tuning stage with supervision from end tasks, whereas we adopt it here as an unsupervised text representation.
} 


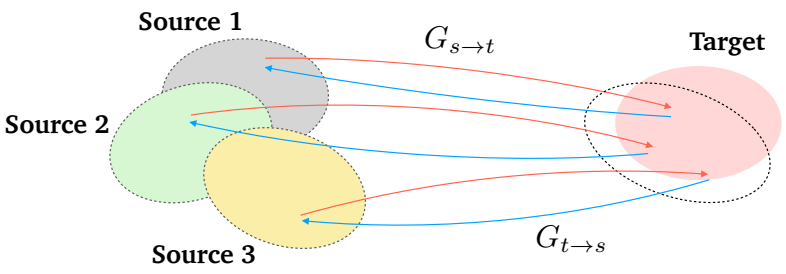

Figure 3: Intermediate domain generation with a CycleGAN.

Figure 3. For that purpose, we introduce a source-to-target generator $G_{s \rightarrow t}$, and train it to generate target representations that aim to fool an adversarial discriminator $D_{t}$. This gives the following GAN loss:

$$
\mathcal{L}_{\text {gan }}^{s \rightarrow t}=\mathbb{E}_{\mathbf{z} \sim Z_{S}} \log \left[D_{t}\left(G_{S \rightarrow t}(\mathbf{z})\right)\right]+\mathbb{E}_{\mathbf{z} \sim Z_{T}} \log \left[1-D_{t}(\mathbf{z})\right]
$$

In order to avoid mode collapse in the generated intermediate domain and encourage the internal structural information of the original example to be preserved, we follow [30] and optimize a cycle-consistency loss which is obtained by recontructing the representation of the original example from the intermediate domain representation. To implement this loss, we introduce a reverse generator from target to source $G_{t \rightarrow s}$, which can be trained using a reverse GAN loss $\mathcal{L}_{\mathrm{adv}}^{t \rightarrow s}$ (this requires an additional discriminator at source side $D_{s}$ ). Then, the cycle-consistency loss can be written as:

$$
\begin{aligned}
\mathcal{L}_{c y c} & =\mathbb{E}_{\mathbf{z} \sim Z_{S}}\left\|G_{t \rightarrow s}\left(G_{s \rightarrow t}(\mathbf{z})\right)-\mathbf{z}\right\|_{1} \\
& +\mathbb{E}_{\mathbf{z} \sim Z_{T}}\left\|G_{s \rightarrow t}\left(G_{t \rightarrow s}(\mathbf{z})\right)-\mathbf{z}\right\|_{1}
\end{aligned}
$$

The above loss function treats all source examples in a training batch equally, while ignoring their varied relevance/importance to the target domain distribution due to the multi-source nature. To cope with this challenge, we explore two instance-level weight assignment mechanisms which operate on each batch: the modelbased curriculum and the model-free curriculum.

Model-based Curriculum. We follow [77] and use an extra source selection network for calculating the weight distribution over examples in a batch. This network takes the generated representation $\left(G_{s \rightarrow t}(\mathbf{z})\right)$ as input, and outputs a weight distribution with a Softmax layer. Denoting $B$ as a batch of encoded examples sampled from the $\mathcal{Z}:\left\{\mathbf{z}_{1}, \mathbf{z}_{2}, \ldots, \mathbf{z}_{|B|}\right\}$, the sample weights can be computed as:

$$
\mathbf{w}=\operatorname{softmax}\left(h_{t}\left(G_{s \rightarrow t}(B)\right)\right)
$$

where $h_{t}$ is the source selection network at target side. We then obtain the curriculum GAN loss $\left(\mathcal{L}_{\text {cgan }}\right)$ as:

$$
\begin{aligned}
\mathcal{L}_{\text {cgan }}^{s \rightarrow t} & =\mathbb{E}_{B \sim \mathcal{Z}_{S}} \frac{1}{|B|} \sum_{\mathbf{z} \in B} w_{z} \log \left[D_{t}\left(G_{s \rightarrow t}(B)\right)\right] \\
& +\mathbb{E}_{\mathbf{z} \sim \mathcal{Z}_{T}} \log \left[1-D_{t}(\mathbf{z})\right]
\end{aligned}
$$

In the ideal case, if the input batch of the source selection network is extremely close to the target distribution, we would expect a uniform weighting. Therefore, we introduce additional inductive bias for training $h_{t}$ by minimizing the KL-divergence between the output distribution and a uniform distribution $(\mathcal{U})$ when the input batch is sampled from the real target space:

$$
\mathcal{L}_{\text {uni }}^{t}=\mathbb{E}_{\mathbf{z} \sim \mathcal{Z}_{T}} \mathrm{KL}\left[h_{t}(\mathbf{z}) \| \mathcal{U}\right]
$$

The formulation of $\mathcal{L}_{c g a n}^{t \rightarrow s}$ and $\mathcal{L}_{u n i}^{s}$ can be adapted in a similar way, using a separate source selection network $h_{s}$.

Model-free Curriculum. Instead of relying on an extra source selection network, we can also compute sample weights directly from outputs of the domain discriminators $\left(D_{t}\right)$, which indeed reflects the proximity of each example to the target domain. This gives us the following model-free weight assignment mechanism:

$$
\mathbf{w}=\operatorname{softmax}\left(\log \left[D_{t}\left(G_{s \rightarrow t}(B)\right)\right]\right)
$$

In this way, examples with a higher probability of being classified as target will be more emphasized in the GAN loss.

\subsection{Task Classifier}

Assuming the source-to-target generation $\left(G_{s \rightarrow t}\right)$ does not change the sentiment label, we can train a transferable sentiment classifier over the generated intermediate domain: $f_{t}: G_{s \rightarrow t}(\mathcal{Z}) \rightarrow \mathcal{Y}$ using labels from the source domains:

$$
\mathcal{L}_{\text {task }}=-\mathbb{E}_{(\mathbf{z}, y) \sim\left(\mathcal{Z}_{S}, \mathcal{Y}_{S}\right)}\left[-\log P\left(y \mid f_{t}\left(G_{s \rightarrow t}(\mathbf{z})\right)\right)\right]
$$

After training, the classifier $f_{t}$ can be directly used in the target domain. To promote sentiment consistency between the generated intermediate representations and their original examples, we further backpropagate the task loss to the source-to-target generator.

\subsection{Joint Learning}

Our final objective is a weighted combination of different losses in the C-CycleGAN framework. ${ }^{2}$ For the model-based curriculum:

$$
\mathcal{L}_{\text {c-cyclegan }}=\mathcal{L}_{\text {cgan }}^{s \rightarrow t}+\mathcal{L}_{\text {cgan }}^{t \rightarrow s}+\mathcal{L}_{\text {cyc }}+\mathcal{L}_{\text {uni }}^{t}+\mathcal{L}_{\text {uni }}^{s}+\mathcal{L}_{\text {task }}
$$

For the model-free curriculum:

$$
\mathcal{L}_{\text {c-cyclegan }}=\mathcal{L}_{\text {cgan }}^{s \rightarrow t}+\mathcal{L}_{\text {cgan }}^{t \rightarrow s}+\mathcal{L}_{\text {cyc }}+\mathcal{L}_{\text {task }}
$$

This objective can be optimized by solving the following minmax game:

$$
f_{t}^{*}=\underset{f_{t}}{\arg \min } \min _{\substack{G_{s \rightarrow t} \\ G_{t \rightarrow s} \\ h_{t}, h_{s}}} \max _{D_{s}, D_{t}} \mathcal{L}_{\text {c-cyclegan }}
$$

\section{EXPERIMENTS}

In this section, we introduce the experimental settings and present results as well as analysis. Our source code will be released.

\subsection{Experimental Settings}

4.1.1 Datasets. We evaluate our approach using two combined datasets of cross-domain sentiment analysis: Reviews-5 [79] and Amazon benchmark [7]. Each dataset contains multiple domains. For each dataset, we create multiple MDA settings by taking each domain as target, and the rest as sources. In addition, we further consider a cross-lingual transfer setting using the Multilingual Amazon Reviews Corpus [8], to validate the generalizability of our approach to a broader family of transfer learning.

\footnotetext{
${ }^{2}$ We omit the weights of each loss term for notation ease.
} 
Table 1: Comparison with the state-of-the-art DA methods on Reviews-5 dataset. All numbers are percentages. The best classwise and average classification accuracies trained on the source domains are emphasized in bold (similar below).

\begin{tabular}{c|c|cccccc}
\hline Standards & Models & Camera & Laptop & Restaurant & Movie1 & Movie2 & Avg \\
\hline \multirow{2}{*}{ Source-only } & Single-best & 68.8 & 62.5 & 64.0 & 76.9 & 75.8 & 69.6 \\
& Source-combined & 69.6 & 71.5 & 68.5 & 77.0 & 76.7 & 72.7 \\
\hline \multirow{2}{*}{ Single-best DA } & DANN [19] & 69.2 & 72.6 & 68.5 & 78.3 & 80.7 & 73.9 \\
& ADDA [66] & 69.4 & 73.2 & 69.6 & 79.1 & 81.5 & 74.6 \\
& DAAN [78] & 69.4 & 73.8 & 71.6 & 79.5 & 82.8 & 75.4 \\
\hline \multirow{2}{*}{ Source-combined DA } & DANN [19] & 67.0 & 73.3 & 68.2 & 77.4 & 80.8 & 73.3 \\
& ADDA [66] & 69.6 & 74.1 & 69.5 & 80.5 & 82.6 & 75.3 \\
& DAAN [78] & 69.4 & 74.6 & 72.4 & 80.2 & 83.2 & 76.0 \\
\hline \multirow{3}{*}{ Multi-source DA } & Autoencoder+MDAN [83] & 65.0 & 59.0 & 64.5 & 60.8 & 52.1 & 60.3 \\
& MDAN (TextCNN) [83] & 68.0 & 72.0 & 71.0 & 77.4 & 78.7 & 73.4 \\
& CMSS [77] & 71.8 & 75.4 & 73.3 & 81.2 & 85.6 & 77.5 \\
& C-CycleGAN (Ours) & $\mathbf{7 3 . 8}$ & $\mathbf{7 6 . 0}$ & $\mathbf{7 6 . 0}$ & $\mathbf{8 2 . 0}$ & $\mathbf{8 7 . 5}$ & $\mathbf{7 9 . 1}$ \\
\hline Oracle & TextCNN & 76.8 & 77.5 & 77.5 & 84.4 & 90.6 & 81.4 \\
\hline
\end{tabular}

Table 2: Comparison with the state-of-the-art DA methods on Reviews-5 dataset using BERT embedding.

\begin{tabular}{c|c|cccccc}
\hline Standards & Models & Camera & Laptop & Restaurant & Movie1 & Movie2 & Avg \\
\hline Source-only & Single-best & 72.3 & 74.5 & 75.4 & 79.4 & 83.1 & 76.9 \\
& Source-combined & 73.6 & 74.8 & 76.8 & 80.1 & 85.7 & 78.2 \\
\hline Multi-source DA & C-CycleGAN (Ours) & $\mathbf{7 6 . 9}$ & $\mathbf{7 8 . 4}$ & $\mathbf{7 9 . 7}$ & $\mathbf{8 3 . 1}$ & $\mathbf{8 8 . 3}$ & $\mathbf{8 1 . 3}$ \\
\hline Oracle & BERT & 78.3 & 79.5 & 81.2 & 85.1 & 90.8 & 83.0 \\
\hline
\end{tabular}

The Reviews-5 dataset [79] includes five domains of customer reviews. Movie1 [45] and Movie2 [54] are movie reviews; Camera [31] contains reviews of digital products such as MP3 players and cameras; Laptop and Restaurant are laptop and restaurant reviews respectively taken from SemEval 2015 Task 12 [79]. The training set sizes are 3,270,1,707, 1,372, 9,162, and 8,113 for Movie1, Movie2, Camera, Laptop and Restaurant, respectively. The test size is 200 for all domains.

The Amazon benchmark dataset [7] contains four domains of product reviews on Amazon: Books, DVD, Kitchen, and Electronics, with training set sizes of $6,465,5,586,7,681$, and 7,945 respectively. The test size is 200 for all domains. This dataset has been preprocessed by the authors into TF-IDF representations, using the 5,000 most frequent unigram and bigram features. Therefore, word order information is unavailable.

The Multilingual Amazon Reviews Corpus [8] is a collection of Amazon reviews from four languages: German, English, French, and fapanese. For each language, there are three domains including Books, DVD, and Music. The training set size and test set size for each domain of each language are 52,000 and 2,000.

4.1.2 Evaluation Metrics. Following [26, 83], we use classification accuracy as metric to evaluate the sentiment classification results. Larger values represent better performances.

\subsubsection{Baselines. We consider the following baselines:}

(1) Source-only, directly training on the source domains and testing on the target domain, which includes two settings: single-best, the best test accuracy on target among all source domains; source-combined, the target accuracy of the model trained on the combined source domain.

(2) Single-source domain adaptation methods, including DANN [19], ADDA [66], and DAAN [78], trained with both single-best and source-combined settings.

(3) Multi-source domain adaptation models, including stateof-the-art approaches MDAN [83], MoE [26], and CMSS [77].

We also report the results of an oracle setting, where the model is both trained and tested on the target domain.

4.1.4 Implementation Details. For the pre-training of text encoder, we use a 2-layer Bidirectional LSTM as encoder and a 1-layer LSTM as decoder. The initial learning rate is 0.00001 with a decay rate of 0.5 every 200 steps. The dimension of word embeddings and hidden states are both set to 256. For experiments with BERT, we use the 12-layer "bert-base-uncased" version due to memory constraints. The weights for $\mathcal{L}_{\text {cgan }}, \mathcal{L}_{\text {cyc }}, \mathcal{L}_{\text {uni }}$, and $\mathcal{L}_{\text {task }}$ are $0.1,1,1$ and 1 , respectively. During decoding, we choose as input between the true previous token and the generated token with a probability of 0.5 of selecting either one. For the Amazon benchmark dataset, we use the original TF-IDF feature vectors as the representation, without further encoding or pre-training. We leverage a 4-layer 
Table 3: Comparison with the state-of-the-art DA methods on Amazon Benchmark dataset.

\begin{tabular}{c|c|ccccc}
\hline Standards & Models & Books & DVD & Kitchen & Electronics & Avg \\
\hline \multirow{2}{*}{ Source-only } & Single-best & 75.4 & 81.3 & 86.5 & 86.5 & 82.4 \\
& Source-combined & 76.5 & 81.6 & 86.7 & 85.3 & 82.5 \\
\hline \multirow{3}{*}{ Single-best DA } & DANN [19] & 76.5 & 77.2 & 83.6 & 84.3 & 80.4 \\
& ADDA [66] & 74.4 & 78.2 & 82.6 & 82.1 & 79.3 \\
& DAAN [78] & 77.2 & 76.8 & 83.5 & 86.5 & 81.0 \\
\hline \multirow{3}{*}{ Source-combined DA } & DANN [19] & 77.9 & 78.9 & 84.9 & 86.4 & 82.0 \\
& ADDA [66] & 76.6 & 77.1 & 82.5 & 82.5 & 79.7 \\
& DAAN [78] & 78.4 & 77.6 & 85.4 & 87.2 & 82.2 \\
\hline \multirow{3}{*}{ Multi-source DA } & MDAN [83] & 78.0 & 85 & 85.3 & 86.3 & 82.5 \\
& MoE [26] & 78.9 & 81.3 & 87.4 & 87.9 & 83.9 \\
& CMSS [77] & 78.1 & 80.2 & 87.2 & 87.2 & 83.2 \\
& C-CycleGAN (Ours) & $\mathbf{8 0 . 3}$ & $\mathbf{8 2 . 2}$ & $\mathbf{8 8 . 9}$ & $\mathbf{8 9 . 1}$ & $\mathbf{8 5 . 1}$ \\
\hline Oracle & TextCNN & 76.7 & 81.3 & 87.1 & 85.2 & 82.6 \\
\hline
\end{tabular}

multi-layer perceptron (MLP) to implement the generator and discriminator of CycleGAN, as well as the sentiment classifier. The initial learning rate is 0.0001 with a decay rate of 0.5 every 100 steps. We use Adam [34] as the optimizer with beta1 of 0.5, beta2 of 0.999 , batch size of 64 , and weight decay of 0.0001 . In the multilingual transfer experiments, we obtain cross-lingual word embeddings by projecting the pre-trained monolingual word embeddings [4] of the 4 languages into English (pivot language) using an unsupervised method [1].

\subsection{Results on Reviews-5 Dataset}

We first evaluate our approach on the dataset of plain textual input: Reviews-5. We perform experiments with each domain as the target and the rest as sources. Table 1 shows the performance of different DA methods and Table 2 shows the extended results using BERT embedding [14]. We have the following observations ${ }^{3}$ :

(1) Without considering domain shift, both source-only settings, i.e. single-best and source-combined, obtain poor accuracy: $69.6 \%$ and $72.7 \%$, around $10 \%$ worse than the oracle $(81.4 \%)$. This motivates the research on domain adaptation.

(2) When directly applying to the MDA task, the single-source DA methods outperform the source-only setting. Since customers' reviews vary a lot across domains, features related to sentiment also vary a lot. Therefore these DA methods that can make the domain gap smaller achieve better results than source-only setting.

(3) Comparing the performances of source-combined and singlebest DA settings, we can find that sometimes naively performing single-source domain adaptation approaches on a combined dataset of different sources could produce worse result (i.e. $73.3 \%$ of DANN) than on a single source domain (i.e. $73.9 \%$ of DANN). This naturally motivates research on multi-source domain adaptation.

(4) Most of the state-of-the-art multi-source domain adaptation methods perform better than single-source domain adaptation methods by considering domain-invariant features and fusing information across all domains. However, MDAN [83], which has

\footnotetext{
${ }^{3}$ The first 5 points are based on Table 1 , and the last point is based on Table 2 .
}

been demonstrated to be effective on Amazon benchmark dataset, performs worse (60.3\% and $73.4 \%)$ than single-best DA settings (e.g. $74.6 \%$ and $75.4 \%$ ). This indicates that some of the previous multisource domain adaptation methods may be only effective on a certain kind of data representation (e.g. bag-of-words or TF-IDF).

(5) C-CycleGAN performs the best (79.1\%) among all adaptation settings. Compared to the best results inside the Source-only, Singlebest DA, Source-dombined DA and other Multi-source DA methods, C-CycleGAN achieves $6.4 \%, 3.7 \%, 3.1 \%$ and $1.6 \%$ performance boost, respectively. These results demonstrate that the proposed C-CycleGAN model can achieve significant better performance compared to state-of-the-art methods. The performance improvements benefit from the advantages of C-CycleGAN. First, an intermediate representation domain is generated with cycle-consistency and sentiment consistency which is closer to the target domain and preserves the annotation information of the source samples. Second, the proposed weighting mechanisms can dynamically assign weights to different source samples, which takes into account the source samples' similarity to the target and enhances the adaptation performance. Finally, the text reconstruction in the pre-trained text encoder minimizes the information loss during the feature encoding process.

(6) BERT embedding performs much better than Bi-LSTM for all the methods, which demonstrates the superiority of BERT in learning pre-trained embeddings. The proposed C-CycleGAN achieves $3.1 \%$ performance gains as compared to the best source-only setting.

\subsection{Results on Amazon Benchmark Dataset}

Table 3 shows the results on the Amazon benchmark dataset, which takes TF-IDF as text representations. We can observe that:

(1) Comparing the performance of source-only (82.5\%) and Oracle $(82.6 \%)$, we can see that the domain gap between sources and target is less than $1 \%$, much smaller than the domain gap of Reviews$5(>10 \%)$. This indicates that the data representation type of the datasets is closely associated with how large the domain gap is. 
Table 4: Comparison with the state-of-the-art DA methods on Multilingual Amazon Reviews Corpus dataset.

\begin{tabular}{|c|c|c|c|c|c|c|c|c|c|}
\hline \multirow{2}{*}{ Standards } & \multirow{2}{*}{ Models } & \multicolumn{4}{|c|}{ German } & \multicolumn{4}{|c|}{ English } \\
\hline & & Books & DVD & Music & Avg & Books & DVD & Music & Avg \\
\hline \multirow{2}{*}{ Source-only } & Single-best & 63.6 & 64.7 & 64.9 & 64.4 & 65.3 & 62.5 & 63.3 & 63.7 \\
\hline & Source-combined & 61.5 & 64.6 & 63.6 & 63.2 & 63.7 & 65.0 & 60.1 & 63.0 \\
\hline Multi-source DA & C-CycleGAN (Ours) & 78.3 & 78.4 & 79.1 & 78.6 & 78.0 & 77.8 & 79.0 & 78.3 \\
\hline Oracle & TextCNN & 83.2 & 89.0 & 88.2 & 86.8 & 85.2 & 85.5 & 81.1 & 83.9 \\
\hline \multirow{2}{*}{ Standards } & \multirow{2}{*}{ Models } & \multicolumn{4}{|c|}{ French } & \multicolumn{4}{|c|}{ Japanese } \\
\hline & & Books & DVD & Music & Avg & Books & DVD & Music & Avg \\
\hline \multirow{2}{*}{ Source-only } & Single-best & 65.3 & 64.3 & 64.2 & 64.6 & 63.5 & 63.5 & 64.8 & 64.0 \\
\hline & Source-combined & 63.6 & 63.0 & 63.4 & 63.3 & 63.7 & 62.7 & 64.0 & 63.4 \\
\hline Multi-source DA & C-CycleGAN (Ours) & 78.6 & 77.6 & 76.9 & 77.7 & 75.2 & 74.9 & 76.8 & 76.2 \\
\hline Oracle & TextCNN & 88.3 & 77.6 & 84.1 & 83.3 & 60.4 & 61.8 & 69.4 & 69.4 \\
\hline
\end{tabular}

Table 5: Ablation study on different components of the proposed C-CycleGAN framework on the Reviews-5 dataset.

\begin{tabular}{l|cccccc}
\hline Models & Camera & Laptop & Restaurant & Movie1 & Movie2 & Avg \\
\hline CycleGAN [94] & 68.7 & 75.4 & 71.6 & 82.5 & 86.7 & 77.0 \\
MDAN [83] + CycleGAN [94] & 70.8 & 75.2 & 71.2 & 79.9 & 86.2 & 76.7 \\
\hline CycleGAN [94]+CMSS [77] & 71.5 & 75.4 & 70.8 & 81.1 & 86.1 & 77.0 \\
C-CycleGAN (model-based) & 72.8 & 75.7 & 73.5 & 81.7 & 87.3 & 78.2 \\
C-CycleGAN (model-free) & $\mathbf{7 3 . 8}$ & $\mathbf{7 6 . 0}$ & $\mathbf{7 6 . 0}$ & $\mathbf{8 2 . 0}$ & $\mathbf{8 7 . 5}$ & $\mathbf{7 9 . 1}$ \\
\hline
\end{tabular}

(2) Several multi-source adaptation methods (e.g. MoE [26]) perform even better than Oracle. This is because that the domain gap is relatively small and multi-source adaptation leverages more information from multiple domains than Oracle, which only has access to the samples from the target. This further indicates the importance of diverse data from different source domains.

(3) The proposed C-CycleGAN has the best performance (85.1\%) among all approaches with $1.2 \%$ and $2.5 \%$ better classification accuracy than MoE and Oracle respectively. Compared to other methods (e.g. MDAN) whose performance fluctuates significantly across datasets (Reviews-5 and Amazon Benchmark datasets), the proposed C-CycleGAN can provide consistent superior performance across datasets.

\subsection{Multilingual Transfer Experiments}

We also perform experiments on the Multilingual Amazon Reviews Corpus. For each category domain (Books, DVD, Music) of each language, we perform adaptation to it with datesets of the same category domain from other languages as sources. Table 4 shows the performance of different adaptation methods. We can observe that:

(1) The proposed C-CycleGAN achieves the best performance of all DA methods across all languages and on all category domains.

(2) In most cases, Oracle gives the best performance; however, in several settings, C-CycleGAN can achieve similar or even better results than the oracle (e.g. 77.6\% and 77.6\% for DVD in French; 76.8\% and $69.4 \%$ for Music in Japanese). This further demonstrate that our framework has a wide range of applicability, not only across different types of data representation, but also across different languages.

\subsection{Ablation Study}

We conduct a series of ablation studies on the Reviews- 5 dataset to demonstrate the improvements of C-CycleGAN over existing state-of-the-art approaches. The results are described in Table 5, where all CycleGANs are performed in a source-combined manner.

First, we investigate whether it is necessary to align the representations before applying CycleGAN. "MDAN + CycleGAN" in Table 5 represents first aligning the encoded representations using MDAN and then applying CycleGAN. Comparing the first two rows in Table 5, we can see applying MDAN before CycleGAN achieves worse performance, which indicates that it is unnecessary to perform additional alignment before CycleGAN. This is probably because extracting the domain-invariant features between the source and target domains might lose some discriminative features in the target domain that are related to sentiment.

Second, we investigate the effectiveness of the proposed modelbased and model-free weighting methods. From the last three rows, we can see that compared to CMSS [77], the proposed model-based and model-free weighting schemes improve accuracy by $1.2 \%$ and $2.1 \%$ respectively. Because CMSS takes the original source samples as input to compute the weights, it cannot reflect the dynamic changing of source samples' weights. The proposed model-based 
Table 6: Ablation study on the influence of cycle-consistency in C-CycleGAN on the Reviews-5 dataset.

\begin{tabular}{l|cccccc}
\hline Models & Camera & Laptop & Restaurant & Movie1 & Movie2 & Avg \\
\hline C-CycleGAN w/o cycle-consistency & 72.2 & 73.1 & 72.0 & 80.3 & 85.1 & 76.5 \\
C-CycleGAN w cycle-consistency & $\mathbf{7 3 . 8}$ & $\mathbf{7 6 . 0}$ & $\mathbf{7 6 . 0}$ & $\mathbf{8 2 . 0}$ & $\mathbf{8 7 . 5}$ & $\mathbf{7 9 . 1}$ \\
\hline
\end{tabular}

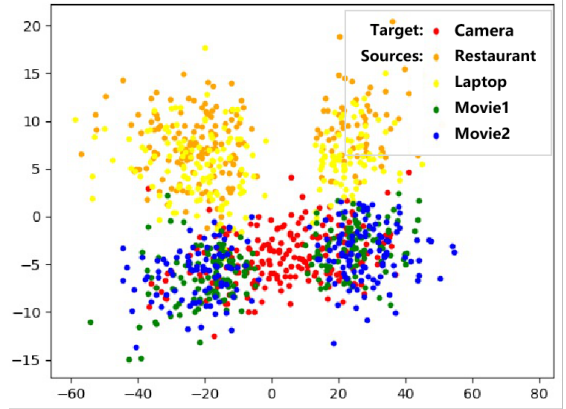

(a) Before adaptation

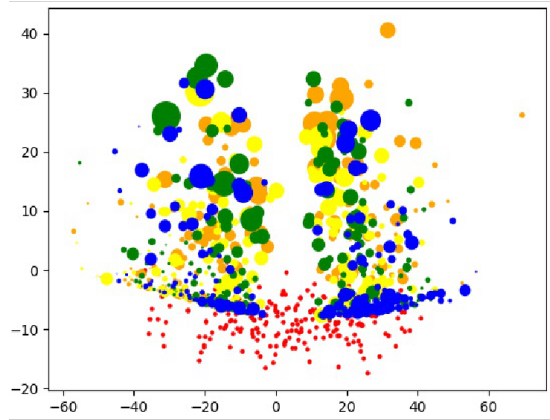

(d) Epoch 100

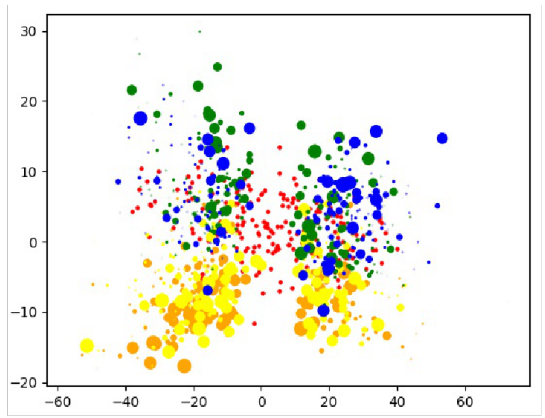

(b) Epoch 10

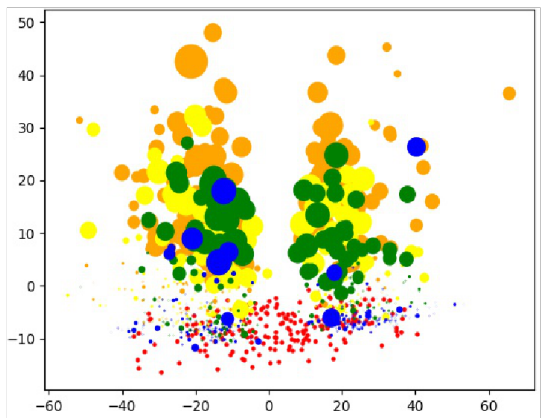

(e) Epoch 200

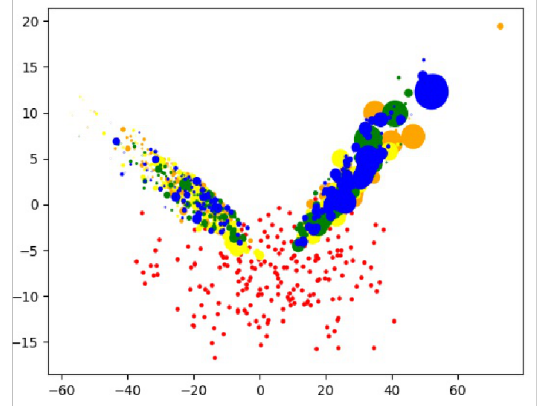

(c) Epoch 50

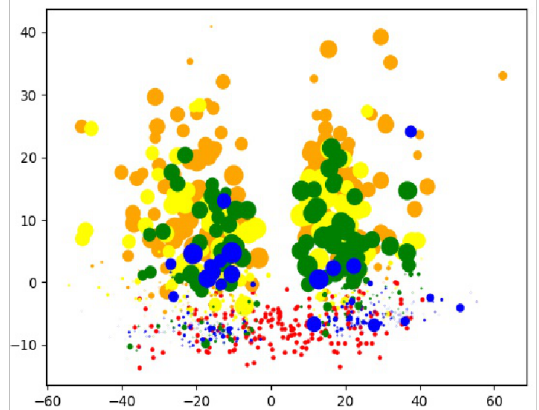

(f) After adaptation

Figure 4: Visualization of feature spaces in different training stages of C-CycleGAN on the Reviews-5 dataset. Target samples are in red, while source samples are in other colors. Point size denotes the similarity of each source sample to the target domain obtained from output of the domain discriminator. For better visualization, smaller points represent samples closer to the target domain.

weighting mechanism is based on the generated intermediate domain, which itself dynamically changes. The model-based method requires an additional network to compute the similarity to the target domain, which not only increase the computation cost, but also takes longer to learn the discriminative patterns between sources and target, before which CycleGAN may learn the wrong patterns.

Finally, we evaluate the influence of cycle-consistency in the proposed C-CycleGAN model. As in [94], we find that standard adversarial procedures without cycle-consistency often lead to the mode collapse problem, where all input representations are mapped to the same output representation and the optimization fails to make progress. The comparison between with and without cycleconsistency in C-CycleGAN on the Reviews-5 dataset is shown in Table 6 . The result comparison (79.1 vs. 76.5 ) clearly demonstrates the effectiveness and necessity of cycle-consistency.

\subsection{Visualization}

In this section, we visualize the features of source and target samples during different training stages of C-CycleGAN.
By using PCA to reduce the dimensionality of samples, we project samples from five domains in Reviews-5 [79] onto a 2-dimensional plane in different stages of training. The visualization results are shown in Figure 4. We can conclude that during the training process, all source domains get closer to the target domain. At the same time, we can see that the samples far from the target domain can be well differentiated by the discriminator, and are assigned with smaller weights (larger points).

Figure 4 (a) and (f) visualize the representation space before and after adaptation correspondingly. We can see that the samples in Movie1 and Movie2 are the closest since they are all about reviews in movies. Movie1 is also closer with Camera and Laptop after adaptation, which is desirable because these domains involve common reviews on image quality or upgrade of electronics. For example, the Camera domain may have reviews like "Picture is clear and easy to carry. Love SONY."; while in Movie1: "Transitions smoothly and the image quality is clean", and in Laptop: "The $4 \mathrm{~K}$ display is so sharp, the slim book is so light in a bag". We can hardly distinguish which domains these reviews belong to without prior information. 

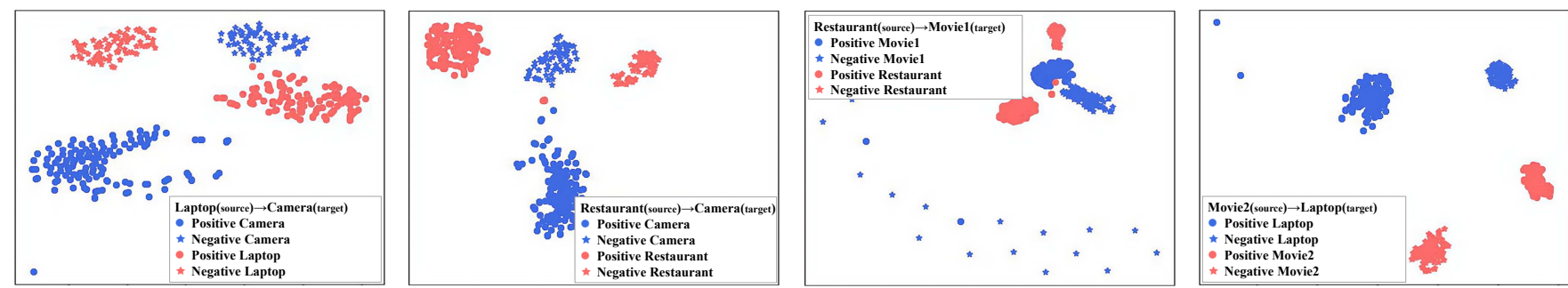

(a) Before adaptation
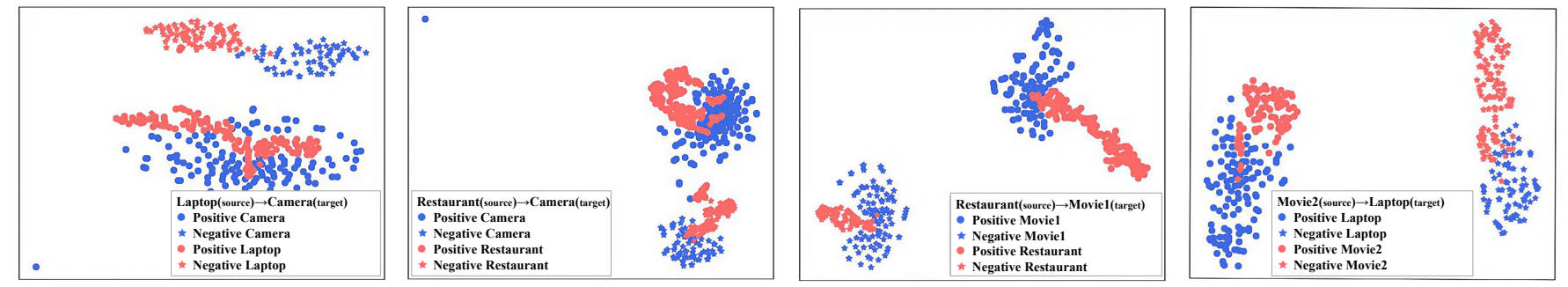

(b) After adaptation

Figure 5: t-SNE visualization of the features before and after adaptation on the Reviews-5 dataset. Red represents source features and Blue represents target features.

We further plot the learned features with t-SNE [43] on four adaptation settings, with the results shown in Figure 5. The top row represents the feature embeddings before adaptation, while the bottom row represents the feature embeddings after adaptation by C-CycleGAN. Red represents source features and Blue represents target features. As we can see, before adaptation, the source samples can be obviously classified but such classifier cannot work well on the target samples; with the proposed C-CycleGAN, source and target features of the same class become more aggregated after adaptation. These observations further demonstrate the effectiveness of C-CycleGAN.

\section{CONCLUSION}

In this paper, we proposed a novel multi-source domain adaptation framework, named curriculum cycle-consistent generative adversarial network (C-CycleGAN), for textual sentiment classification. C-CycleGAN contains three main component: pre-trained text encoder for encoding text instances into a latent continuous representation space with minimal information loss; intermediate domain generator with curriculum instance-level adaptation considering the importance of different source samples; and task classifier to perform the final sentiment classification. The generated intermediate domain bridges the domain gap between the source and target domains, while preserving the sentiment semantics. The proposed dynamic model-based and model-free weighting mechanisms can assign higher weights to the source samples that are closer to the target domain. Further, C-CycleGAN does not require prior domain labels of source samples, which makes it more practical in realworld scenarios. Extensive experiments on multiple benchmark datasets demonstrate that C-CycleGAN significantly outperforms existing state-of-the-art DA methods. In future studies, we plan to construct a large-scale textual dataset with more fine-grained sentiment categories and extend our framework to corresponding
MDA tasks. We will explore multi-modal domain adaptation by jointly modeling multiple modalities, such as image and text.

\section{ACKNOWLEDGMENTS}

This work is supported by Berkeley DeepDrive, the Major Project for New Generation of AI Grant (No. 2018AAA0100403), the National Natural Science Foundation of China (Nos. 61701273, 61876094, U1933114), Natural Science Foundation of Tianjin, China (Nos. 20JCJQJC00020, 18JCYBJC15400, 18ZXZNGX00110), and the Fundamental Research Funds for the Central Universities.

\section{REFERENCES}

[1] Mikel Artetxe, Gorka Labaka, and Eneko Agirre. 2017. Learning bilingual word embeddings with (almost) no bilingual data. In Annual Meeting of the Association for Computational Linguistics. 451-462.

[2] Shai Ben-David, John Blitzer, Koby Crammer, Alex Kulesza, Fernando Pereira, and Jennifer Wortman Vaughan. 2010. A theory of learning from different domains. Machine Learning 79, 1-2 (2010), 151-175.

[3] Rhys Biddle, Aditya Joshi, Shaowu Liu, Cecile Paris, and Guandong Xu. 2020. Leveraging Sentiment Distributions to Distinguish Figurative From Literal Health Reports on Twitter. In The Web Conference. 1217-1227.

[4] Piotr Bojanowski, Edouard Grave, Armand Joulin, and Tomas Mikolov. 2017. Enriching Word Vectors with Subword Information. Transactions of the Association for Computational Linguistics 5 (2017), 135-146.

[5] Fabio M Carlucci, Antonio D'Innocente, Silvia Bucci, Barbara Caputo, and Tatiana Tommasi. 2019. Domain generalization by solving jigsaw puzzles. In IEEE Conference on Computer Vision and Pattern Recognition. 2229-2238.

[6] Rita Chattopadhyay, Qian Sun, Wei Fan, Ian Davidson, Sethuraman Panchanathan, and Jieping Ye. 2012. Multisource domain adaptation and its application to early detection of fatigue. ACM Transactions on Knowledge Discovery from Data 6, 4 (2012), 18.

[7] Minmin Chen, Zhixiang Xu, Kilian Q Weinberger, and Fei Sha. 2012. Marginalized denoising autoencoders for domain adaptation. In International Conference on Machine Learning. 1627-1634.

[8] Xilun Chen, Ahmed Hassan Awadallah, Hany Hassan, Wei Wang, and Claire Cardie. 2019. Multi-Source Cross-Lingual Model Transfer: Learning What to Share. In Annual Meeting of the Association for Computational Linguistics. 30983112.

[9] Xi Chen, Hang Li, Chenyi Zhou, Xue Liu, Di Wu, and Gregory Dudek. 2020. FiDo: Ubiquitous Fine-Grained WiFi-based Localization for Unlabelled Users via Domain Adaptation. In The Web Conference. 23-33. 
[10] Yi-Hsin Chen, Wei-Yu Chen, Yu-Ting Chen, Bo-Cheng Tsai, Yu-Chiang Frank Wang, and Min Sun. 2017. No more discrimination: Cross city adaptation of road scene segmenters. In IEEE International Conference on Computer Vision. 1992-2001.

[11] Zhenpeng Chen, Sheng Shen, Ziniu Hu, Xuan Lu, Qiaozhu Mei, and Xuanzhe Liu. 2019. Emoji-powered representation learning for cross-lingual sentiment classification. In International World Wide Web Conference. 251-262.

[12] Kyunghyun Cho, Bart van Merriënboer, Caglar Gulcehre, Dzmitry Bahdanau, Fethi Bougares, Holger Schwenk, and Yoshua Bengio. 2014. Learning Phrase Representations using RNN Encoder-Decoder for Statistical Machine Translation. In Conference on Empirical Methods in Natural Language Processing. 1724-1734.

[13] Jan Deriu, Aurelien Lucchi, Valeria De Luca, Aliaksei Severyn, Simon Müller Mark Cieliebak, Thomas Hofmann, and Martin Jaggi. 2017. Leveraging large amounts of weakly supervised data for multi-language sentiment classification In International World Wide Web Conference. 1045-1052.

[14] Jacob Devlin, Ming-Wei Chang, Kenton Lee, and Kristina Toutanova. 2019. BERT Pre-training of Deep Bidirectional Transformers for Language Understanding. In Annual Conference of the North American Chapter of the Association for Computational Linguistics. 4171-4186.

[15] Zhengming Ding, Ming Shao, and Yun Fu. 2018. Incomplete multisource transfer learning. IEEE Transactions on Neural Networks and Learning Systems 29, 2 (2018) 310-323.

[16] Li Dong, Furu Wei, Chuanqi Tan, Duyu Tang, Ming Zhou, and Ke Xu. 2014 Adaptive recursive neural network for target-dependent twitter sentiment classification. In Annual Meeting of the Association for Computational Linguistics. 49-54.

[17] Lixin Duan, Dong Xu, and Shih-Fu Chang. 2012. Exploiting web images for event recognition in consumer videos: A multiple source domain adaptation approach In IEEE Conference on Computer Vision and Pattern Recognition. 1338-1345.

[18] Lixin Duan, Dong Xu, and Ivor Wai-Hung Tsang. 2012. Domain adaptation from multiple sources: A domain-dependent regularization approach. IEEE Transactions on Neural Networks and Learning Systems 23, 3 (2012), 504-518.

[19] Yaroslav Ganin, Evgeniya Ustinova, Hana Ajakan, Pascal Germain, Hugo Larochelle, François Laviolette, Mario Marchand, and Victor Lempitsky. 2016. Domain-adversarial training of neural networks. fournal of Machine Learning Research 17, 1 (2016), 2096-2030.

[20] Spiros V Georgakopoulos, Sotiris K Tasoulis, Aristidis G Vrahatis, and Vassilis P Plagianakos. 2018. Convolutional neural networks for toxic comment classification. In Hellenic Conference on Artificial Intelligence. 1-6.

[21] Muhammad Ghifary, W Bastiaan Kleijn, Mengjie Zhang, and David Balduzzi. 2015. Domain generalization for object recognition with multi-task autoencoders. In IEEE International Conference on Computer Vision. 2551-2559.

[22] Muhammad Ghifary, W Bastiaan Kleijn, Mengjie Zhang, David Balduzzi, and Wen Li. 2016. Deep reconstruction-classification networks for unsupervised domain adaptation. In European Conference on Computer Vision. 597-613.

[23] Lin Gong, Benjamin Haines, and Hongning Wang. 2017. Clustered model adaption for personalized sentiment analysis. In International World Wide Web Conference. 937-946.

[24] Lin Gong and Hongning Wang. 2018. When sentiment analysis meets social network: A holistic user behavior modeling in opinionated data. In ACM SIGKDD International Conference on Knowledge Discovery and Data Mining. 1455-1464.

[25] Ian Goodfellow, Jean Pouget-Abadie, Mehdi Mirza, Bing Xu, David Warde-Farley, Sherjil Ozair, Aaron Courville, and Yoshua Bengio. 2014. Generative adversarial nets. In Advances in Neural Information Processing Systems. 2672-2680.

[26] Jiang Guo, Darsh Shah, and Regina Barzilay. 2018. Multi-Source Domain Adaptation with Mixture of Experts. In Conference on Empirical Methods on Natural Language Processing. 4694-4703.

[27] Sepp Hochreiter and Jürgen Schmidhuber. 1997. Long short-term memory. Neural Computation 9, 8 (1997), 1735-1780.

[28] Judy Hoffman, Mehryar Mohri, and Ningshan Zhang. 2018. Algorithms and theory for multiple-source adaptation. In Advances in Neural Information Processing Systems. 8246-8256.

[29] Judy Hoffman, Eric Tzeng, Taesung Park, Jun-Yan Zhu, Phillip Isola, Kate Saenko, Alexei A Efros, and Trevor Darrell. 2018. CyCADA: Cycle-Consistent Adversarial Domain Adaptation. In International Conference on Machine Learning. 1994-2003.

[30] Judy Hoffman, Dequan Wang, Fisher Yu, and Trevor Darrell. 2016. Fcns in the wild: Pixel-level adversarial and constraint-based adaptation. arXiv preprint arXiv:1612.02649 (2016).

[31] Minqing Hu and Bing Liu. 2004. Mining and summarizing customer reviews. In ACM SIGKDD International Conference on Knowledge Discovery and Data Mining. $168-177$.

[32] Haoshuo Huang, Qixing Huang, and Philipp Krahenbuhl. 2018. Domain transfer through deep activation matching. In European Conference on Computer Vision. 590-605

[33] Guoliang Kang, Lu Jiang, Yi Yang, and Alexander G Hauptmann. 2019. Contrastive Adaptation Network for Unsupervised Domain Adaptation. In IEEE Conference on Computer Vision and Pattern Recognition. 4893-4902.
[34] Diederik P Kingma and Jimmy Ba. 2015. Adam: A method for stochastic optimization. In International Conference on Learning Representations.

[35] Svetlana Kiritchenko, Xiaodan Zhu, and Saif M Mohammad. 2014. Sentiment analysis of short informal texts. Fournal of Artificial Intelligence Research 50 (2014), 723-762.

[36] Wouter Marco Kouw and Marco Loog. 2019. A review of domain adaptation without target labels. IEEE Transactions on Pattern Analysis and Machine Intelligence (2019).

[37] Shanu Kumar, Shubham Atreja, Anjali Singh, and Mohit Jain. 2019. Adversarial adaptation of scene graph models for understanding civic issues. In International World Wide Web Conference. 2943-2949.

[38] Ming-Yu Liu and Oncel Tuzel. 2016. Coupled generative adversarial networks. In Advances in Neural Information Processing Systems. 469-477.

[39] Qiao Liu, Haibin Zhang, Yifu Zeng, Ziqi Huang, and Zufeng Wu. 2018. Content attention model for aspect based sentiment analysis. In International World Wide Web Conference. 1023-1032.

[40] Ruijun Liu, Yuqian Shi, Changjiang Ji, and Ming Jia. 2019. A survey of sentiment analysis based on transfer learning. IEEE Access 7 (2019), 85401-85412.

[41] Mingsheng Long, Yue Cao, Jianmin Wang, and Michael Jordan. 2015. Learning transferable features with deep adaptation networks. In International Conference on Machine Learning. 97-105.

[42] Washington Luiz, Felipe Viegas, Rafael Alencar, Fernando Mourão, Thiago Salles, Dárlinton Carvalho, Marcos Andre Gonçalves, and Leonardo Rocha. 2018. A feature-oriented sentiment rating for mobile app reviews. In International World Wide Web Conference. 1909-1918.

[43] Laurens van der Maaten and Geoffrey Hinton. 2008. Visualizing data using t-SNE. fournal of Machine Learning Research 9, 11 (2008), 2579-2605.

[44] Saif Mohammad, Svetlana Kiritchenko, and Xiaodan Zhu. 2013. NRC-Canada: Building the State-of-the-Art in Sentiment Analysis of Tweets. In International Workshop on Semantic Evaluation (SemEval). 321-327.

[45] Bo Pang and Lillian Lee. 2005. Seeing Stars: Exploiting Class Relationships for Sentiment Categorization with Respect to Rating Scales. In Annual Meeting of the Association for Computational Linguistics. 115-124.

[46] Bo Pang and Lillian Lee. 2008. Opinion mining and sentiment analysis. Foundations and Trends in Information Retrieval 2, 1-2 (2008), 1-135.

[47] Vishal M Patel, Raghuraman Gopalan, Ruonan Li, and Rama Chellappa. 2015. Visual domain adaptation: A survey of recent advances. IEEE Signal Processing Magazine 32, 3 (2015), 53-69.

[48] Xingchao Peng, Qinxun Bai, Xide Xia, Zijun Huang, Kate Saenko, and Bo Wang. 2019. Moment Matching for Multi-Source Domain Adaptation. In IEEE International Conference on Computer Vision. 1406-1415.

[49] Qiao Qian, Bo Tian, Minlie Huang, Yang Liu, Xuan Zhu, and Xiaoyan Zhu. 2015. Learning tag embeddings and tag-specific composition functions in recursive neural network. In Annual Meeting of the Association for Computational Linguistics. 1365-1374.

[50] Ievgen Redko, Nicolas Courty, Rémi Flamary, and Devis Tuia. 2019. Optimal transport for multi-source domain adaptation under target shift. In International Conference on Artificial Intelligence and Statistics. 849-858.

[51] Jian Shen, Yanru Qu, Weinan Zhang, and Yong Yu. 2017. Wasserstein distance guided representation learning for domain adaptation. arXiv preprint arXiv:1707.01217 (2017).

[52] Ashish Shrivastava, Tomas Pfister, Oncel Tuzel, Josh Susskind, Wenda Wang, and Russ Webb. 2017. Learning from simulated and unsupervised images through adversarial training. In IEEE Conference on Computer Vision and Pattern Recognition. $2242-2251$.

[53] Richard Socher, Jeffrey Pennington, Eric H Huang, Andrew Y Ng, and Christopher D Manning. 2011. Semi-supervised recursive autoencoders for predicting sentiment distributions. In Conference on Empirical Methods on Natural Language Processing. 151-161.

[54] Richard Socher, Alex Perelygin, Jean Wu, Jason Chuang, Christopher D Manning, Andrew Y Ng, and Christopher Potts. 2013. Recursive deep models for semantic compositionality over a sentiment treebank. In Conference on Empirical Methods in Natural Language Processing. 1631-1642.

[55] Baochen Sun, Jiashi Feng, and Kate Saenko. 2016. Return of frustratingly easy domain adaptation. In AAAI Conference on Artificial Intelligence. 2058-2065.

[56] Baochen Sun, Jiashi Feng, and Kate Saenko. 2017. Correlation alignment for unsupervised domain adaptation. In Domain Adaptation in Computer Vision Applications. 153-171.

[57] Qian Sun, Rita Chattopadhyay, Sethuraman Panchanathan, and Jieping Ye. 2011. A two-stage weighting framework for multi-source domain adaptation. In Advances in Neural Information Processing Systems. 505-513.

[58] Shiliang Sun, Honglei Shi, and Yuanbin Wu. 2015. A survey of multi-source domain adaptation. Information Fusion 24 (2015), 84-92.

[59] Shi-Liang Sun and Hong-Lei Shi. 2013. Bayesian multi-source domain adaptation. In International Conference on Machine Learning and Cybernetics, Vol. 1. 24-28.

[60] Yu Sun, Eric Tzeng, Trevor Darrell, and Alexei A Efros. 2019. Unsupervised Domain Adaptation through Self-Supervision. arXiv preprint arXiv:1909.11825 (2019) 
[61] Kai Sheng Tai, Richard Socher, and Christopher D Manning. 2015. Improved Semantic Representations From Tree-Structured Long Short-Term Memory Networks. In Annual Meeting of the Association for Computational Linguistics. 15561566.

[62] Duyu Tang, Bing Qin, and Ting Liu. 2015. Document modeling with gated recurrent neural network for sentiment classification. In Conference on Empirical Methods on Natural Language Processing. 1422-1432.

[63] Antonio Torralba and Alexei A Efros. 2011. Unbiased look at dataset bias. In IEEE Conference on Computer Vision and Pattern Recognition. 1521-1528.

[64] Yi-Hsuan Tsai, Wei-Chih Hung, Samuel Schulter, Kihyuk Sohn, Ming-Hsuan Yang, and Manmohan Chandraker. 2018. Learning to adapt structured output space for semantic segmentation. In IEEE Conference on Computer Vision and Pattern Recognition. 7472-7481.

[65] Eric Tzeng, Judy Hoffman, Trevor Darrell, and Kate Saenko. 2015. Simultaneous deep transfer across domains and tasks. In IEEE International Conference on Computer Vision. 4068-4076.

[66] Eric Tzeng, Judy Hoffman, Kate Saenko, and Trevor Darrell. 2017. Adversarial discriminative domain adaptation. In IEEE Conference on Computer Vision and Pattern Recognition. 2962-2971.

[67] Thuy-Trang Vu, Dinh Phung, and Gholamreza Haffari. 2020. Effective Unsupervised Domain Adaptation with Adversarially Trained Language Models. In Conference on Empirical Methods in Natural Language Processing.

[68] Junxiang Wang and Liang Zhao. 2018. Multi-instance domain adaptation for vaccine adverse event detection. In International World Wide Web Conference. 97-106.

[69] Yequan Wang, Aixin Sun, Jialong Han, Ying Liu, and Xiaoyan Zhu. 2018. Sentiment analysis by capsules. In International World Wide Web Conference. 11651174 .

[70] Yequan Wang, Aixin Sun, Minlie Huang, and Xiaoyan Zhu. 2019. Aspect-level sentiment analysis using as-capsules. In International World Wide Web Conference. 2033-2044.

[71] Man Wu, Shirui Pan, Chuan Zhou, Xiaojun Chang, and Xingquan Zhu. 2020 Unsupervised Domain Adaptive Graph Convolutional Networks. In The Web Conference. 1457-1467.

[72] Dongbo Xi, Fuzhen Zhuang, Ganbin Zhou, Xiaohu Cheng, Fen Lin, and Qing He. 2020. Domain Adaptation with Category Attention Network for Deep Sentiment Analysis. In The Web Conference. 3133-3139.

[73] Jiaolong Xu, Liang Xiao, and Antonio M López. 2019. Self-Supervised Domain Adaptation for Computer Vision Tasks. IEEE Access 7 (2019), 156694-156706.

[74] Ruijia Xu, Ziliang Chen, Wangmeng Zuo, Junjie Yan, and Liang Lin. 2018. Deep cocktail network: Multi-source unsupervised domain adaptation with category shift. In IEEE Conference on Computer Vision and Pattern Recognition. 3964-3973.

[75] Zhijie Xu and Shiliang Sun. 2012. Multi-source transfer learning with multi-view adaboost. In International Conference on Neural Information Processing. 332-339.

[76] Ashima Yadav and Dinesh Kumar Vishwakarma. 2020. Sentiment analysis using deep learning architectures: a review. Artificial Intelligence Review 53, 6 (2020), 4335-4385.

[77] Luyu Yang, Yogesh Balaji, Ser-Nam Lim, and Abhinav Shrivastava. 2020. Curriculum Manager for Source Selection in Multi-Source Domain Adaptation. In European Conference on Computer Vision. 608-624.

[78] Chaohui Yu, Jindong Wang, Yiqiang Chen, and Meiyu Huang. 2019. Transfer learning with dynamic adversarial adaptation network. In IEEE International Conference on Data Mining. 778-786.

[79] Jianfei Yu and Jing Jiang. 2016. Learning sentence embeddings with auxiliary tasks for cross-domain sentiment classification. In Conference on Empirical Methods in Natural Language Processing. 236-246.

[80] Xiangyu Yue, Yang Zhang, Sicheng Zhao, Alberto Sangiovanni-Vincentelli, Kurt Keutzer, and Boqing Gong. 2019. Domain randomization and pyramid consistency: Simulation-to-real generalization without accessing target domain data. In IEEE International Conference on Computer Vision. 2100-2110.

[81] Lei Zhang, Shuai Wang, and Bing Liu. 2018. Deep learning for sentiment analysis: A survey. Wiley Interdisciplinary Reviews: Data Mining and Knowledge Discovery 8, 4 (2018), e1253.

[82] Zufan Zhang, Yang Zou, and Chenquan Gan. 2018. Textual sentiment analysis via three different attention convolutional neural networks and cross-modality consistent regression. Neurocomputing 275 (2018), 1407-1415.

[83] Han Zhao, Shanghang Zhang, Guanhang Wu, José MF Moura, Joao P Costeira, and Geoffrey J Gordon. 2018. Adversarial multiple source domain adaptation. In Advances in Neural Information Processing Systems. 8568-8579.

[84] Sicheng Zhao, Guiguang Ding, Yue Gao, Xin Zhao, Youbao Tang, Jungong Han Hongxun Yao, and Qingming Huang. 2020. Discrete probability distribution prediction of image emotions with shared sparse learning. IEEE Transactions on Affective Computing 11, 4 (2020), 574-587.

[85] Sicheng Zhao, Bo Li, Colorado Reed, Pengfei Xu, and Kurt Keutzer. 2020. Multisource Domain Adaptation in the Deep Learning Era: A Systematic Survey. arXiv preprint arXiv:2002.12169 (2020).

[86] Sicheng Zhao, Bo Li, Xiangyu Yue, Yang Gu, Pengfei Xu, Runbo Hu, Hua Chai, and Kurt Keutzer. 2019. Multi-source Domain Adaptation for Semantic Segmentation.
In Advances in Neural Information Processing Systems. 7285-7298.

[87] Sicheng Zhao, Chuang Lin, Pengfei Xu, Sendong Zhao, Yuchen Guo, Ravi Krishna, Guiguang Ding, and Kurt Keutzer. 2019. CycleEmotionGAN: Emotional Semantic Consistency Preserved CycleGAN for Adapting Image Emotions. In AAAI Conference on Artificial Intelligence. 2620-2627.

[88] Sicheng Zhao, Yunsheng Ma, Yang Gu, Jufeng Yang, Tengfei Xing, Pengfei Xu, Runbo Hu, Hua Chai, and Kurt Keutzer. 2020. An End-to-End visual-audio attention network for emotion recognition in user-generated videos. In $A A A I$ Conference on Artificial Intelligence. 303-311.

[89] Sicheng Zhao, Guangzhi Wang, Shanghang Zhang, Yang Gu, Yaxian Li, Zhichao Song, Pengfei Xu, Runbo Hu, Hua Chai, and Kurt Keutzer. 2020. Multi-source Distilling Domain Adaptation. In AAAI Conference on Artificial Intelligence. 1297512983.

[90] Sicheng Zhao, Hongxun Yao, Yue Gao, Rongrong Ji, Wenlong Xie, Xiaolei Jiang, and Tat-Seng Chua. 2016. Predicting personalized emotion perceptions of social images. In ACM International Conference on Multimedia. 1385-1394.

[91] Sicheng Zhao, Hongxun Yao, and Xiaolei Jiang. 2015. Predicting continuous probability distribution of image emotions in valence-arousal space. In ACM International Conference on Multimedia. 879-882.

[92] Sicheng Zhao, Xiangyu Yue, Shanghang Zhang, Bo Li, Han Zhao, Bichen Wu, Ravi Krishna, Joseph E Gonzalez, Alberto L Sangiovanni-Vincentelli, Sanjit A Seshia, and Kurt Keutzer. 2020. A Review of Single-Source Deep Unsupervised Visual Domain Adaptation. IEEE Transactions on Neural Networks and Learning Systems (2020).

[93] Sicheng Zhao, Xin Zhao, Guiguang Ding, and Kurt Keutzer. 2018. EmotionGAN: unsupervised domain adaptation for learning discrete probability distributions of image emotions. In ACM International Conference on Multimedia. 1319-1327.

[94] Jun-Yan Zhu, Taesung Park, Phillip Isola, and Alexei A Efros. 2017. Unpaired Image-To-Image Translation Using Cycle-Consistent Adversarial Networks. In IEEE International Conference on Computer Vision. 2223-2232.

[95] Junbao Zhuo, Shuhui Wang, Weigang Zhang, and Qingming Huang. 2017. Deep Unsupervised Convolutional Domain Adaptation. In ACM International Conference on Multimedia. 261-269. 